CAPÍTULO 6

\title{
APROVISIONAMIENTO DE LA LECHE CRUDA EN LA CADENA DE SUMINISTROS LÁCTEOS EN EL DEPARTAMENTO DE SUCRE
}





\section{Introducción}

En la actualidad, el crecimiento económico y el aumento del flujo de bienes y recursos conllevan a las empresas a realizar sus operaciones con mayor eficiencia para no convertirse en los eslabones débiles de las cadenas de suministros a las cuales pertenecen.

Dentro de la cadena de suministros de la leche o de los productos lácteos, la cual presenta una estructura de eslabones que inicia a partir de las granjas productoras o ganaderías, continuando con los centros de acopio y empresas industriales procesadoras, y por último los clientes, (Mojica, Trujillo, Castellanos, \& Bernal, 2007). El proceso de aprovisionamiento de la leche cruda como materia prima, y todas las empresas involucradas en este, se consideran como uno de los puntos más relevantes de esta cadena, (Meneses, Estrada, Chantré, \& López, 2015), el cual que se ve afectado por las demoras en los tiempos de recolección que atentan contra las propiedades microbiológicas y físico-químicas del producto si no se brindan las condiciones necesarias para su conservación, (Castillo \& Bermeo, 2013).

La leche es considerada uno de los productos alimenticios más importantes, debido a sus grandes aportes nutricionales y a la gran variedad de subproductos que se pueden derivar de esta, constituyéndose como un bien de primera necesidad en la canasta familiar, que a diferencia de otras cadenas, existe una gran relevancia en cuanto a la calidad de los productos derivados y la seguridad alimentaria por ser altamente perecederos, (Jaramillo \& Areiza, 2012).

En este sentido, en el departamento de Sucre, la producción de leche asume un papel relevante en la actividad agropecuaria y agroindustrial, en donde la contribución del sector de la agricultura, ganadería, caza, silvicultura y pesca al producto interno bruto (PIB), según cifras del DANE en el 2016 es del 10,8\%, lo que evidencia una participación elevada del 
sector en la dinámica de la economía de la región, siendo superada solo por los servicios sociales, comunales y personales.

Consecuencia de ello, se refleja en el uso de la tierra, en donde del suelo apto para agricultura que corresponde al 51,4\% del territorio, solo se emplea para este fin un 10,6\%. Sin embargo, para la actividad ganadera, el suelo apto es del 20,1\% y se emplea para este propósito un 53,4\%. Lo que conlleva a que el suelo apto para agricultura, es usado en la ganadería de tipo extensivo que predomina en Sucre. Figura 26

\begin{tabular}{|c|c|c|}
\hline AGRICULTURA & DEPARTAMENTO & GANADERIA \\
\hline $27,1 \%$ & $\begin{array}{l}\text { BOLÍVAR } \\
2.665 .495 \text { ha }\end{array}$ & $15,8 \%$ \\
\hline $50,8 \%$ & $\begin{array}{l}\text { CÓRDOBA } \\
2.499 .858 \text { ha }\end{array}$ & $11,5 \%$ \\
\hline $10,1 \%$ & $\begin{array}{c}\text { MAGDALENA } \\
2.314 .438 \mathrm{ha}\end{array}$ & $9,1 \%$ \\
\hline $39,4 \%$ & $\begin{array}{l}\text { CESAR } \\
2.256 .550 \text { ha }\end{array}$ & $13,1 \%$ \\
\hline $\begin{array}{r}10,8 \% \\
4,8 \% \square\end{array}$ & $\begin{array}{l}\text { LA GUAJIRA } \\
2.061 .936 \text { ha }\end{array}$ & $\begin{array}{l}26,4 \% \\
21,4 \%\end{array}$ \\
\hline $51,4 \%$ & $\begin{array}{l}\text { SUCRE } \\
1.071 .860 \text { ha }\end{array}$ & $20,1 \%$ \\
\hline $56,3 \%$ & $\begin{array}{l}\text { ATLÁNTICO } \\
331.159 \text { ha }\end{array}$ & $9,4 \%$ \\
\hline No disponible I & $\begin{array}{l}\text { SAN ANDRÉS Y PROV. } \\
4.972 \mathrm{ha}\end{array}$ & $\begin{array}{l}0,2 \% \\
\text { | No disponible }\end{array}$ \\
\hline & Suelo apto $\square$ Suelo usa & \\
\hline
\end{tabular}

Figura 26. Conflicto del uso del suelo rural.

Fuente: Unidad de Planificación Rural Agropecuaria. Corporación PBA.

La explotación principal de leche proviene del ganado bovino, con una predominación del sistema doble propósito (obtención de carne y leche), debido que, se ajusta a las condiciones territoriales de la región utilizando de forma intensiva los recursos abundantes que en este caso son las tierras de bajo valor y la mano de obra familiar, (Jaramillo \& Areiza, 2012).

En Sucre, el proceso de aprovisionamiento que comprende la planificación, ejecución y control del flujo eficiente y rentable de la leche 
cruda para su posterior procesamiento y comercialización directa o por intermediarios mediante la logística de distribución (Govindan, Soleimani, \& Kannan, Reverse logistics and closed-loop supply chain: A comprehensive review to explore the future, 2014), es un proceso que se ejecuta según la experiencia de las acopiadoras para programar la ruta de los vehículos que no cuentan con sistemas de refrigeración alguno, por lo cual no se ofrece una recolección eficiente que proteja el producto de agentes externos, sumado a las demoras en el proceso de recolección y los costos asociados, (Berna, 2011).

De acuerdo con lo anterior, en este capítulo se esbozará la cadena de suministros de lácteos de Sucre haciendo énfasis en el funcionamiento del proceso de aprovisionamiento de la leche cruda en este departamento, siendo el proceso de transporte uno de los aspectos más críticos a considerar, si se quiere aportar a la eficiencia de la cadena y su competitividad.

\section{Metodología}

Esta investigación de tipo descriptiva, se realizó en el departamento de Sucre, tomando como población a los productores, transportadores y acopiadores-procesadores pertenecientes al proceso de aprovisionamiento de la leche cruda, de los cuales se extrajo una muestra tomando como punto de partida las empresas matriculadas ante Cámara de Comercio de Sincelejo en el año 2016, con registro sanitario del INVIMA del mismo año y pertenecientes a los municipios que representan aproximadamente el 70\% de la producción de leche del departamento.

Para este proceso de caracterización de la red de aprovisionamiento, se diseñaron tres instrumentos de recolección de información para cada una de las actividades que hacen parte de este segmento de la cadena, siendo tres instrumentos dirigidos a: centros de acopio y procesadoras de leche, ganaderías y unidades productoras de leche y por último a los vehículos utilizados para la recolección y el transporte.

Posteriormente, para el análisis de cada uno de los eslabones se consideraron los siguientes aspectos:

Producción primaria: se tuvo en cuenta, únicamente el volumen de leche vendida directamente a los procesadores y centros de acopio del departamento de Sucre, que representa el 34\% del total de la leche vendida, (Red Nacional de Agencias de Desarrollo Local, 2013). 
Transporte: se delimitó al transporte terrestre realizado en el siguiente esquema, procesadoras/acopiadoras - Unidades productivas-Procesadoras/ acopiadoras, con vehículos propios o contratados directamente por las unidades productoras/acopiadoras. No se tuvo en cuenta los intermediarios.

Empresas procesadoras/acopiadoras: se focalizaron aquellas empresas legalizadas ante cámara de comercio, que presentaban registros sanitarios según el INVIMA, que compran directamente a las unidades productivas y se encargan de la organización de sus rutas para la recolección de la leche con vehículos propios o contratados.

Esta investigación se desarrolló en 3 fases metodológicas, de la siguiente manera:

1. Revisión y recolección de la información: se obtuvo información con datos de los entes gubernamentales, los cuales fueron verificados y actualizados. Además de datos suministrados por anteriores estudios sobre la temática. Identificando los agentes que intervienen, las variables, parámetros y restricciones propias de la red, teniendo en cuenta tres actores fundamentales, los cuales son: los centros de acopios o procesadores de leche, las ganaderías o unidades productoras, y los vehículos utilizados para la recolección y el transporte.

2. Diseño y aplicación de los instrumentos de medición: para el desarrollo de esta investigación se diseñó, validó y aplicó tres encuestas dirigidas a los agentes mencionados anteriormente, con el fin de recolectar la información en campo relacionada con el manejo de la leche cruda, almacenamiento, equipos utilizados, e información general de los sitios de estudio.

3. Sistematización y análisis de la información: una vez diligenciadas las encuestas y los perfiles sanitarios se diseñaron planillas en Excel para sistematizar la información y hacer los análisis respectivos.

\section{Resultados}

\section{Producción de leche en Sucre}

El departamento de Sucre ubicado en la Región Caribe Colombiana, muestra una gran vocación agropecuaria, la cual aportó al segundo trimestre del 2016 el 10,8\% del PIB departamental. La obtención de la leche se realiza a través del sistema doble propósito mediante la cual se consolida 
leche y carne. Este sistema es propicio para el departamento, debido que se ajusta a las condiciones del territorio, siendo el ganado bovino la fuente de explotación principal, (Jaramillo \& Areiza, 2012).

Para el año 2016, Sucre reportó un total de 862.008 cabezas de ganado ocupando el puesto número 12 en el país, con una producción aproximadamente 170.994.379 litros de leche/año, (Instituto Colombiano Agropecuario - ICA, 2016). (Ver Figura 27). Del total de bovinos, aproximadamente el $66,2 \%$ son dedicadas al sistema doble propósito, quedando por encima de la producción de carne la cual representa el 32,4\% del total de bovinos, y aún más por encima de la producción especializada, la cual registra solo el 1,4\%, (Red Nacional de Agencias de Desarrollo Local, 2013).

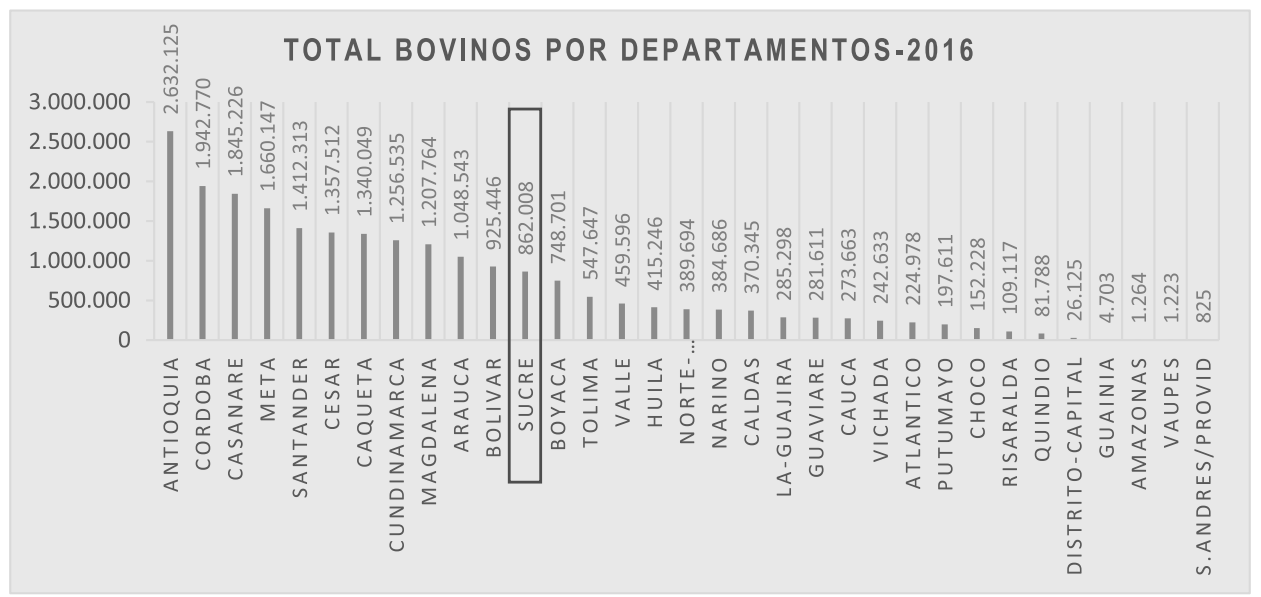

Figura 27. Número total de Bovinos por departamentos en Colombia, Censo Pecuario Nacional 2016.

Fuente: adaptado de Instituto Colombiano Agropecuario - ICA (2016). 
Aprovisionamiento de la leche cruda en la cadena de suministros lácteos en el departamento de sucre

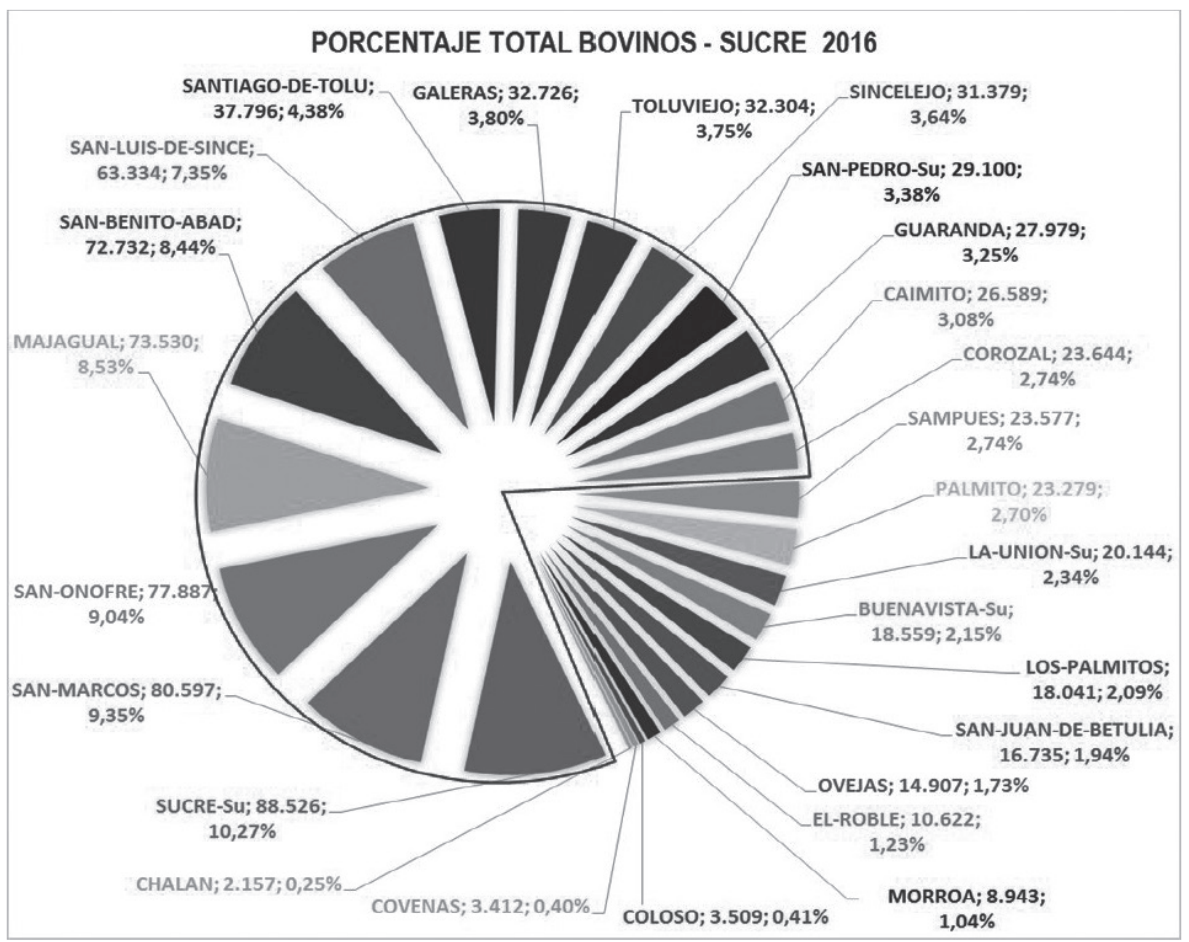

Figura 28. Porcentaje y número total de bovinos por municipios del departamento de Sucre.

Fuente: Adaptado de (Instituto Colombiano Agropecuario - ICA, 2016).

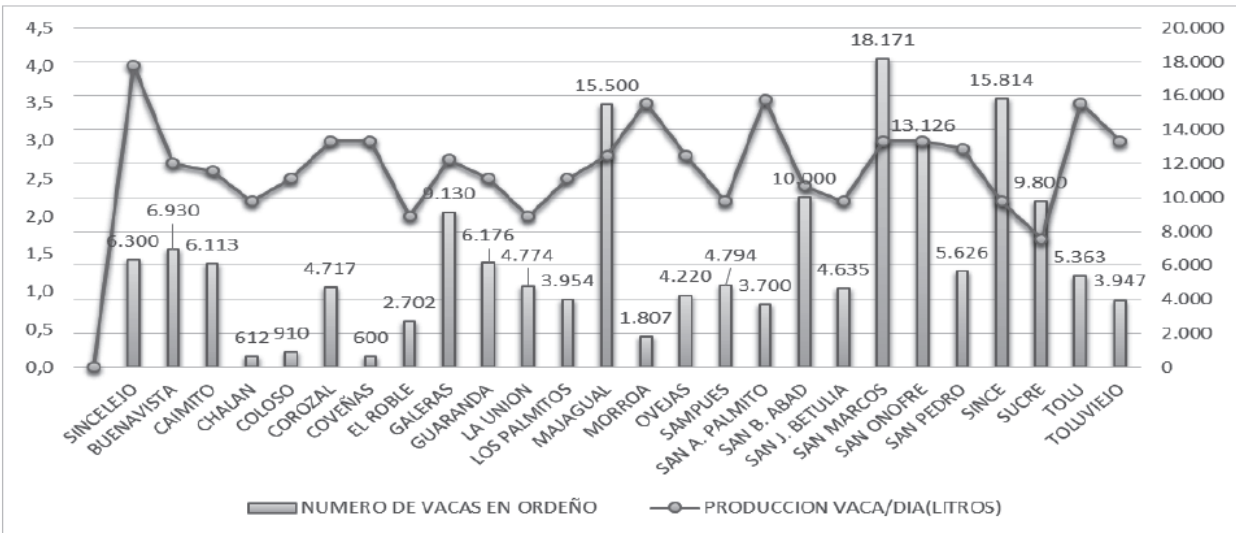

Figura 29. No de vacas en ordeño de Sucre a 2015 y promedio de producción lechera.

Fuente: Secretaria de Desarrollo Económico y Medio Ambiente de Sucre. 
Los municipios con mayor producción de leche a 2015 son San Marcos, que es el mayor productor de los 26 municipios con 54.513 Lt/ día y representa el $11,91 \%$ de la producción departamental. Majagual que produce $43.400 \mathrm{Lt} /$ día con el 9,48\%, San Onofre 39.378 Lt/día con el 8,60\%, Sincé 34.791 Lt/día con el 7,602\%. (Secretaria de Desarrollo Económico y Medio Ambiente, 2016). Toda esta producción es obtenida tal como se mencionó anteriormente, mediante el sistema doble propósito en un $99,63 \%$.

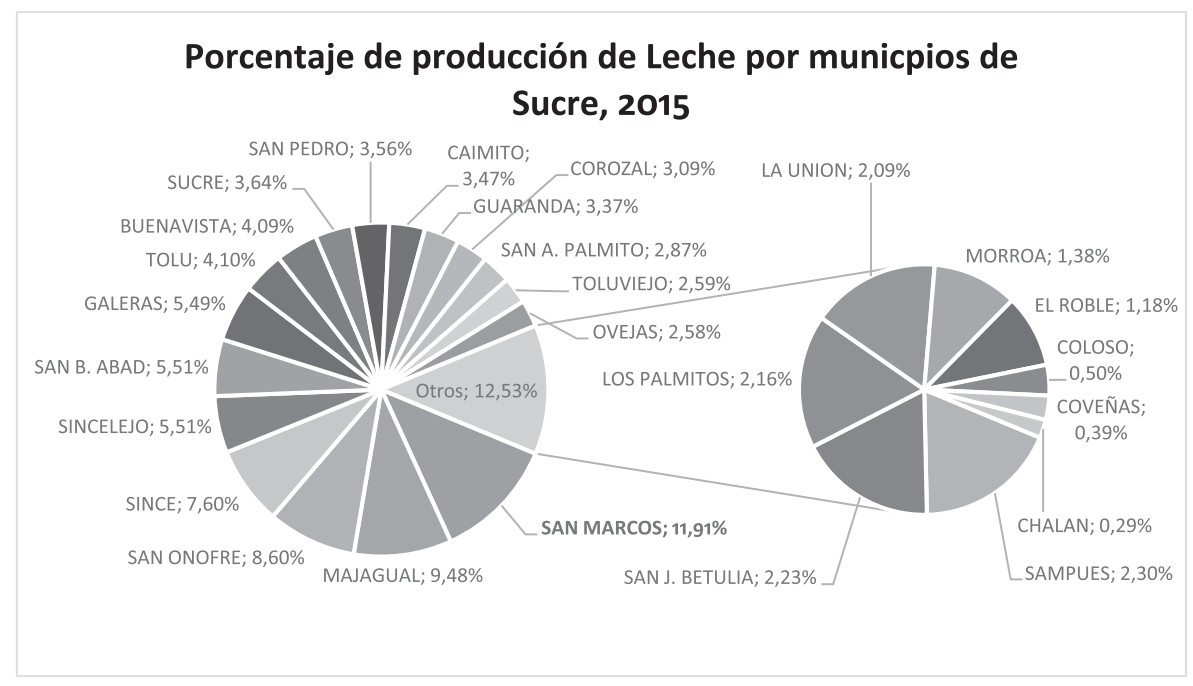

Figura 30. Porcentaje de producción de leche diaria por municipios del Departamento de Sucre, 2015.

Fuente: Adaptado a partir de Secretaria de Desarrollo Económico y Medio Ambiente (2016).

\section{Cadena de suministros de lácteos en Sucre}

La cadena de suministros de lácteos en el mundo

La producción de leche en el contexto internacional se enmarca en dos tipos de metodologías o técnicas que se aplican para su obtención, las cuales son: las producciones industrializadas en las que se aplican avances tecnológicos y razas especializadas tipo lecheras y la producción a pequeña escala que emplea razas variadas por lo general dedicadas al doble propósito (carne y leche), (Jaramillo \& Areiza, 2012). 
De igual modo, la dinámica del mercado internacional está dividida en dos grupos de países: los países altamente desarrollados los cuales necesitan amplios subsidios debido a las condiciones agroclimáticas que generan altos costos, y los países con bajos costos de producción que poseen condiciones agroclimáticas muy favorables para la producción y cuentan con la infraestructura necesaria, (Ministerio de Agricultura y Desarrollo Rural, 2010).

Según datos de la FAO (2015), la producción total de leche a nivel mundial correspondiente al año 2014 fue de 804,5 millones de toneladas métricas, (Comité Nacional Sistema Producto Bovino Leche, 2015). En el marco de américa Latina y el Caribe, conformada por países con potencial lechero como Brasil, Argentina, México, Colombia y Ecuador; presentó el año 2011 una producción de 68 millones de toneladas para Sudamérica, 14.4 millones para América Central (incluyendo México) y 1.9 millones para la región del Caribe.

En este sentido, "La cadena láctea de América Latina y el Caribe, entendida como la sucesión de segmentos que arrancan con la provisión de insumos, la producción primaria, la industrialización y distribución de productos, hasta llegar a millones de consumidores nacionales y extranjeros, es un sector de gran importancia económica, social y territorial en casi todos los países de la región”, (FAO \& FEPALE, 2012, pág. 8).

\section{La cadena de la leche en Colombia}

La cadena de suministros de lácteos es un sector fundamental y estratégico para el desarrollo del país, debido al grado de importancia en la generación de empleo, la seguridad alimentaria y la productividad. Este sector, promedia una participación de $1.5 \%$ del PIB nacional, dividido en un $1,1 \%$ originado en la producción primaria de leche, y el restante 0,4\% en la elaboración de productos lácteos, (Consejo Nacional Lácteo, 2011).

Según Proexport (2011, p. 4), "Colombia se ha posicionado como el cuarto productor de leche con un volumen aproximado de 6.500 millones de toneladas por año en América Latina, superado sólo por Brasil, México y Argentina. A nivel mundial, Colombia ocupa una posición privilegiada al ubicarse en el lugar número 15 dentro del ranking total de productores de leche, con unas ventas anuales de US\$2.862 millones". 
La cadena de suministros de la leche en Colombia, denominada como un "conjunto de actividades que se articulan técnica y económicamente, desde el inicio de la producción y elaboración de un producto agropecuario, hasta su comercialización final”, (FUNDACIÓN ALPINA, 2012, pág. 9), está articulada por seis (6) eslabones que se muestran a continuación, (Consejo Nacional Lácteo, 2011). (Ver Figura 31).

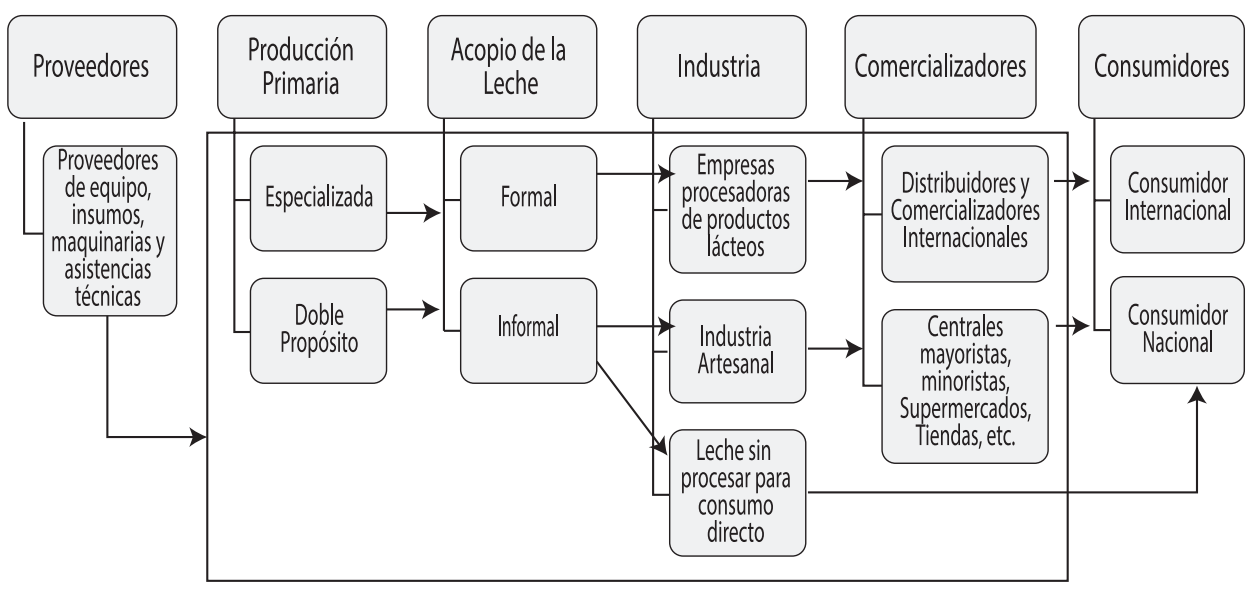

Figura 31. Representación Gráfica los eslabones de la Cadena de Suministro Láctea.

Fuente: Adaptada de Consejo Nacional Lácteo (2011).

Los actores que hacen parte de esta cadena son los proveedores, ganaderos, cruderos, intermediarios, productores, comercializadores, distribuidores, transportistas y consumidores. Los cuales presentan la siguiente relación: los proveedores impactan los eslabones de producción, acopio e industria; los intermediarios por otro lado, hacen parte proceso de acopio y comercialización. De igual modo, los productores o transformadores, están ligados directamente a dos eslabones, el de acopio e industria. Otros actores que tienen más de una relación con los eslabones, son los transportadores, quienes hacen parte de la cadena en los eslabones de acopio y comercialización. (Figura 26). 
Aprovisionamiento de la leche cruda en la cadena de suministros lácteos en el departamento de sucre

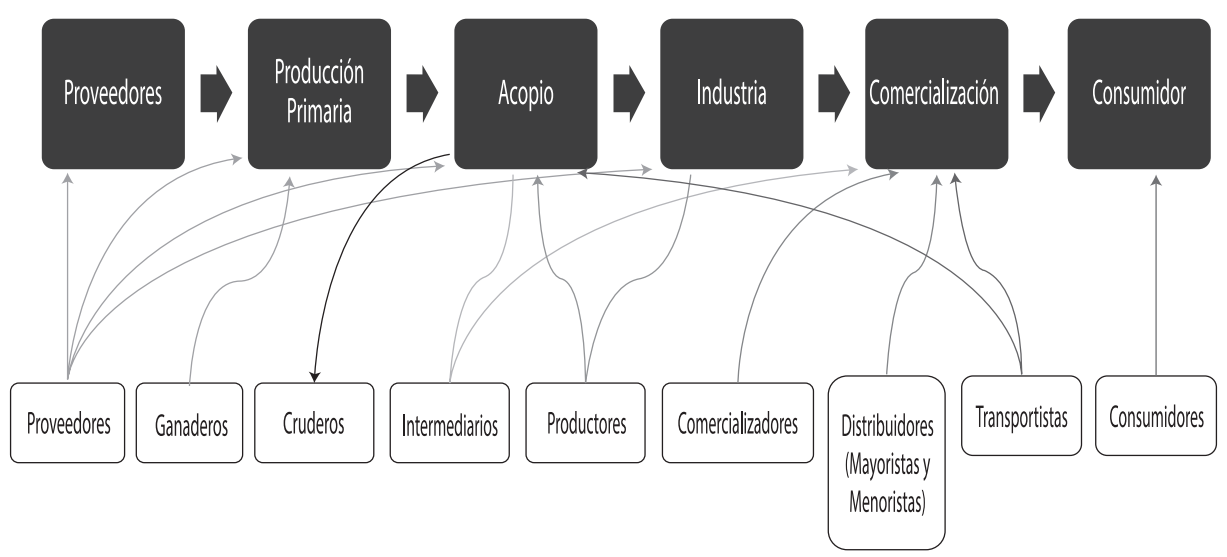

Figura 32. Relación de actores en los eslabones de la cadena de suministros lácteos en Colombia.

Fuente: elaboración propia.

\section{La cadena láctea en Sucre}

La cadena de suministros de lácteos del departamento de Sucre, solo presenta cinco eslabones, debido que el número de centros de acopio son escasos, y el acopio es realizado en las empresas que elaboran productos lácteos. (Figura 27).

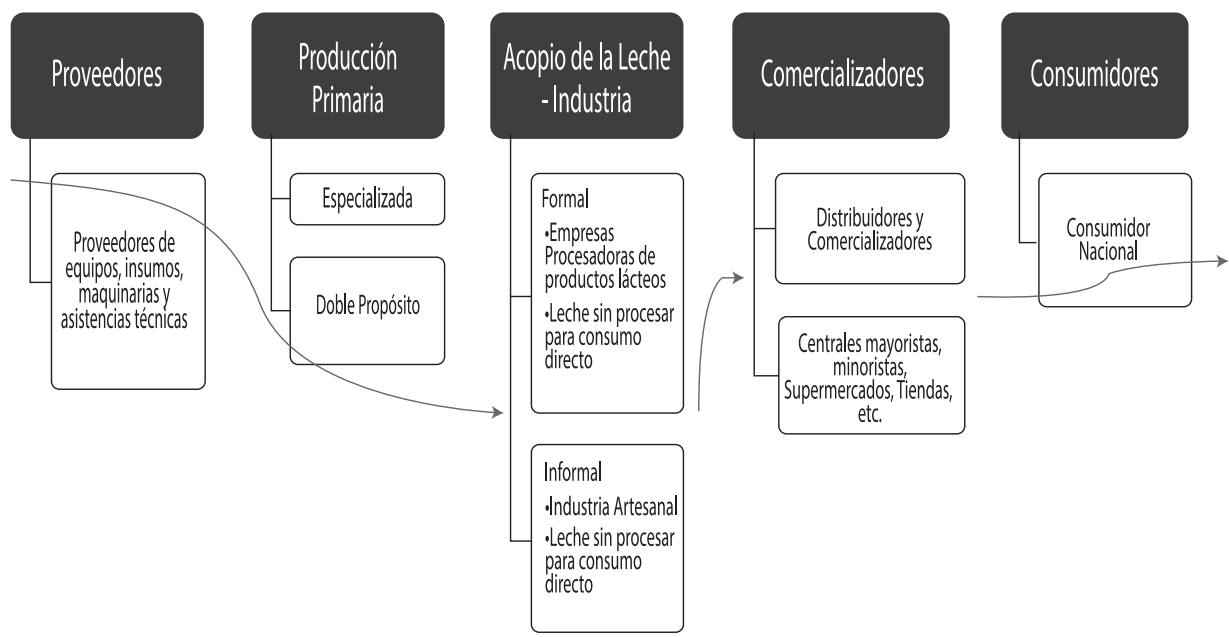

Figura 33. Cadena de Suministros de Lácteos del Departamento de Sucre.

Fuente: elaboración propia. 
A continuación, se detallan los eslabones que conforman la cadena de suministros de lácteos de Sucre.

\section{Proveedores}

Los proveedores constituyen un eslabón transversal a los eslabones de producción primaria, acopio-industria y comercialización, y está conformado por todas aquellas empresas o personas que brindan todos los suministros requeridos para poder obtener productos lácteos.

Producción Primaria

En el departamento de Sucre, el eslabón de producción está conformado por pequeños, medianos y grandes ganaderos que producen la leche principalmente bajo el sistema doble propósito, que representa el $89,39 \%$ de la forma de producción, mientras que el sistema especializada solo alcanza el 0,61\%. El 9,98\% restante representa la obtención solo de carne, (Secretaria de Desarrollo Económico y Medio Ambiente, 2016).

Acopio-Industria

En Sucre, se evidencian cinco actores en este eslabón los cuales son transportadores, cruderos, acopiadores-procesadores, procesadores artesanales y por último, los intermediarios. Cada uno, cumpliendo un rol diferente en la cadena.

Intermediarios, quienes a 2012 obtenían el 62\% del total de la leche dedicada a la venta, (Red Nacional de Agencias de Desarrollo Local, 2013). Estos agentes, se encargan de recolectar la leche directamente en las ganaderías para su posterior venta las empresas. Lo que genera un aumento en el costo de los productos a lo largo de la cadena.

- Cruderos, quienes comercializan la leche cruda directamente a las personas.

- Transportadores, encargados de recolectar la leche en las unidades productivas y entregadas e las acopiadoras-procesadoras. Generalmente son vehículos propios de las empresas o con contrato directo.

- Procesadores-Acopiadores, los cuales se encargan de acopiar la leche cruda y realizar los procesos de transformación pertinente para la obtención de derivados lácteos. Uno de los puntos más 
críticos de estos actores, es el cumplimiento de los aspectos sanitarios, debido que según datos del INVIMA a enero 25 de 2016, solo hay 46 empresas procesadoras pertenecientes a 14 municipios de los 26 del departamento que cumplen con los permisos.

- Productores Artesanales, que realizan la producción de suero y queso costeño a mínima escala y es comercializada a tiendas y a clientes directos.

\section{Consumidores.}

Este eslabón está constituido por el mercado nacional, regional y local, debido a la falta de procesos de exportación de estos productos.

Condiciones de la red de aprovisionamiento de la leche cruda en el departamento de Sucre

El proceso de aprovisionamiento consiste en la articulación de actividades para la planeación, ejecución y control de insumos y materias primas para elaborar un producto, (Govindan, Soleimani, \& Kannan, 2014). Por ende, dentro de la cadena de suministros de lácteos, el aprovisionamiento encierra las actividades de suministro y acopio de las materias primas y en caso esencial de la leche cruda; la planeación y ejecución de las entregas desde la red de proveedores, a la unidad acopiadora o procesadora, debido a limitaciones dadas entre otros factores, por aspectos de transporte, es un aspecto fundamental a considerar en esta cadena, (Chauhan, Eremeev, Romanova, Servakh, \& Woeginger, 2005).

El transporte es considerado uno de los aspectos más críticos en la cadena de suministro, el cual requiere ser un servicio de calidad en términos de seguridad, regularidad, oportunidad, entregas a tiempo y costos, y más aún en la cadena láctea, debido que la leche cruda es un producto altamente perecedero, (Jiménez \& Hernández, 2002). Y sumado a esto, los costos promedios de transporte para la producción lechera en ganaderías colombianas con sistemas doble propósitos a 2009 representan en promedio el 12\% del total de costos de producción, (CONPES, 2010).

Uno de los factores que generan los costos elevados del transporte, que en un $80 \%$ se realiza por el modo carretero, son los fletes derivados del mal estado de las vías rurales y urbanas, que se reflejan en deterioro 
de los vehículos y la demora en la entrega de los productos, (Ministerio de transporte, 2010).

En Sucre, los vehículos empleados no cuentan con sistemas de refrigeración y emplean diferentes tipos de recipientes para transportar la leche que no cumplen con las especificaciones higiénicas establecidas en el Decreto 3075 de 1997 del ministerio de Salud. Los vehículos empleados son: camiones sencillos, camionetas tipo estaca, motos y hasta vehículos de tracción animal. Entre los recipientes utilizados están: tanques plásticos de 200 litros, tanques plásticos de 5 galones fabricados para el empaque de aceite, recipientes de acero inoxidable o aluminio de 40 litros y 20 litros. Transportando aproximadamente entre 1200 litros por vehículo. (Ver Figura 34 Figura 35).

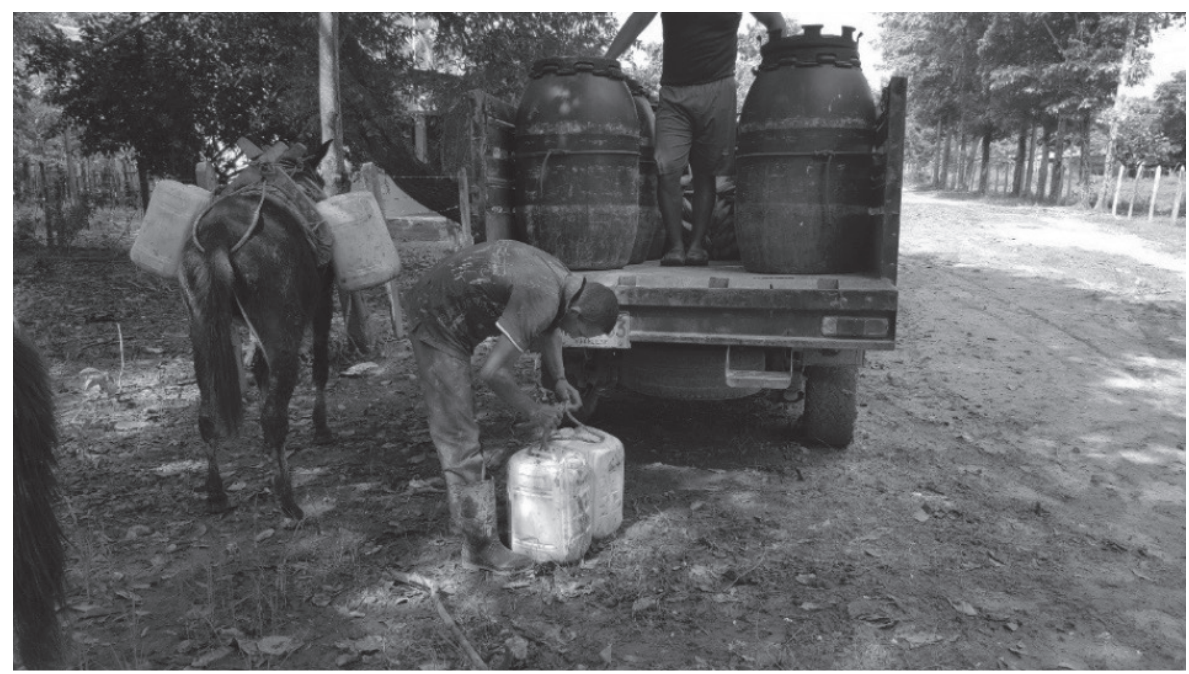

Figura 34. Transporte de leche cruda en vehículos de tracción animal.

Fuente: elaboración propia. 


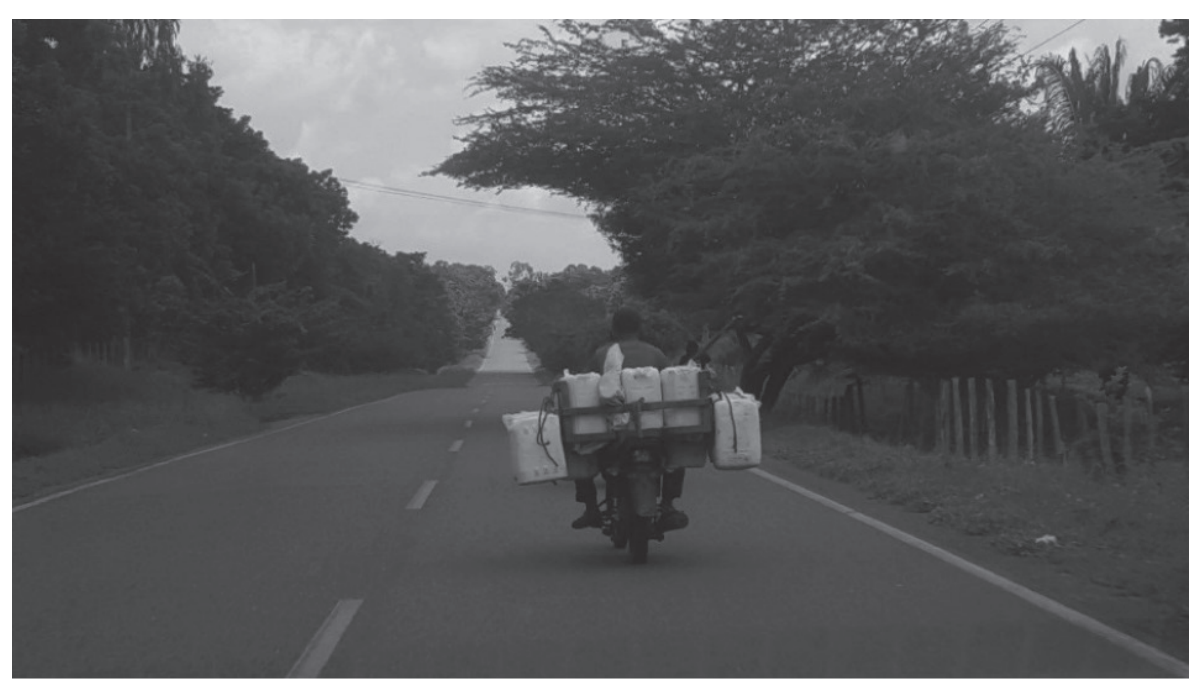

Figura 35. Transporte en moto de leche cruda.

Fuente: elaboración propia.

Las ganaderías, por el uso extensivo de la tierra, se ubican generalmente en zonas rurales alejadas e incluso separadas por Ciénegas y otros cuerpos de agua (subregiones de San Jorge y Mojana), lo que aumenta el tiempo que demora la leche sin refrigeración, generando el crecimiento acelerado de bacterias, (Celis \& Juárez, 2009). Esto, está ocasionando que la leche cruda presente alcohol y altos porcentajes de acidez, (Martínez \& Gómez, Compositional and Hygienic Quality of Raw Molk Received in Dairies in Sucre, Colombia, 2013).

\section{Conclusiones}

En Sucre, la cadena de suministros de lácteos no presenta una estructura definida que evidencie la correlación de actores en cada uno de los eslabones de la cadena, lo que la convierte en un sector poco competitivo. Por lo cual, con este proceso investigativo se definió la estructura formal de la cadena de suministros de lácteos del departamento de Sucre, entendida como el conjunto de actores que se articulan de manera técnica y económica desde la provisión de insumos hasta a entrega del producto final, en cinco eslabones: proveedores, producción primaria, acopio/industria, comercialización y consumidores. 
Dentro del proceso logístico de aprovisionamiento de la leche cruda como materia prima, el transporte se considera un punto crítico en la cadena, el cual no es realizado bajo las condiciones establecidas en los decretos nacionales y se incide directamente en la afectación de la calidad de la leche en los aspectos higiénico-sanitarios, debido a la proliferación bacteriana que se genera.

La leche debe ser transportada a una temperatura a $4{ }^{\circ} \mathrm{C}+/-2^{\circ} \mathrm{C}$, y en caso de mantenerse a temperatura ambiente, según el National Dairy Council, la regla establece que este producto puede estar sin refrigerar por alrededor de dos horas. Sin embargo, en Sucre, los vehículos empleados para este fin, no cuentan con sistemas de refrigeración y generalmente el ruteo tarda un promedio entre cuatro y cinco horas.

Finalmente, con el desarrollo de esta investigación se brinda una base científica para la toma de decisiones de actores estatales sobre cifras y datos concisos, que permiten diseñar estrategias aterrizadas para mejorar aspectos de asociatividad, producción, comercialización, distribución y aprovisionamiento a lo largo de la cadena. 


\section{Referencias Bibliográficas}

Aguilera-Díaz, M. M. (2005). La economía del departamento de Sucre: ganadería y sector público. . Documentos de Trabajo Sobre Economía Regional y Urbana, No. 63.

Batt, P. J., \& Purchase, S. (2004). Managing collaboration within networks and relationships. Industrial marketing management, 33(3), 169-174.

Berna, L. (2011). Modelo de ruteo para entregas de mercancías a clientes por terceros en Sector Retail.

Betts, T., \& Tadisina, S. K. (2009). Supply chain agility, collaboration, and performance: how do they relate? , 1-22. POMS 20th Annual Conference, 1-22.

Bititci, U. S., \& Mokadam, M. (2010). Development of a collaborative supply chain model. In 17th International Conference of the European Operations Management Association, 1-10.

Bohorquez, N., Buitrago, A., Joya, M., Montaña, X., \& Rivera, H. (2012). Análisis Estructural de Sectores Estratégicos: Sector productos Lácteos. (Documento de Investigación; 135). Bogotá: Editorial Universidad del Rosario.

Bowersox, D. J., Closs, D. J., \& Stank, T. P. (2003.). How to master cross-enterprise collaboration. Supply Chain Management Review,. SUPPLY CHAIN MANAGEMENT REVIEW, 7(4), 18-27.

Calderòn, M. E., \& Orjuela, J. A. (2004). Competitividad en la Cadena Agroindustrial láctea. Universidad Distrital Francisco José de Caldas, Bogotà, CO, No. 338.177 Cl46c.

Cao, M., Vonderembse, M. A., Zhang, Q., \& Ragu-Nathan, T. S. (2010). Supply chain collaboration: conceptualisation and instrument development. International Journal of Production Research, 48(22), 6613-6635.

Castillo, H., \& Bermeo, J. (2013). Propuestas de Sistemas Inteligentes para la Implementación de un Modelo de Gestión Triple A en la Cadena de Suministros de Centros de Acopio de Leche cruda en la Provincia de Azuay. Cuenca - Ecuador: Universidad Plitecnica Salesiana.

Celis, M., \& Juárez, D. (2009). Microbiología de la Leche. Editorial de la Universidad Tecnológica Nacional - edUTecNe.

Chauhan, S., Eremeev, A., Romanova, A., Servakh, V., \& Woeginger, G. (2005). Approximation of the supply sheduling problem. Operational Reseach Letters $\mathrm{N}^{\circ} 33,249-254$. 
Chen, L. X., \& Li, J. (2008). Retailer's order policy for perishable products with demand information updating. Mathematics in Practice and Theory, 1, pp. 34-40.

Chen, W., Li, J., \& Jin, X. (15 de April de 2016). The replenishment policy of agri-products with stochastic demand in integrated agricultural supply chains. Expert Systems with Applications, 48, 55-66.

Cho, M., Song, M., Comuzzi, M., \& Yoo, S. (2017). Evaluating the effect of best practices for business process redesign: An evidence-based approach based on process mining techniques. Decision Support Systems, 104, 92-103.

Chounta, I. A., Hecking, T., Hoppe, H. U., \& Avouris, N. (2014). Two Make a Network: Using Graphs to Assess the Quality of Collaboration of Dyads. Collaboration and Technology. Springer, 53-66.

Comité Nacional Sistema Producto Bovino Leche. (2015). Situation de la Lechería a Nivel Mundial. Mexico: US Dairy Export Council.

CONPES. (2010). 3675. Política Nacional para Mejorar la Competitivad del Sector Lácteo Colombiano. Bogotá: Consejo Nacional de Política Económica y Social. Departamento Nacional de Planeación.

Consejo Nacional Lácteo. (2011). Propuesta de Valor Cadena Láctea Colombiana. Programa de Transformación Productiva (PTP). Bogotá.

DANE. (2012). Informe de Coyuntura Ecónomica Regional Departamento de Sucre. Sincelejo: Banco de la República.

DANE. (06 de Noviembre de 2015). Tercer Censo Nacional Agropecuario. Décima entrega resultados 2014- cifras preliminares. Bogotá, Colombia: Departamento Administrativo Nacional de Estadística.

Dania, W. A., Xing, K., \& Amer, Y. (2018). Collaboration behavioural factors for sustainable agri-food supply chains: A systematic review. Journal of Cleaner Production, 186, 851-864.

Daugherty, P. J., Richey, R. G., Roath, A. S., \& Genchev, S. E. (2006). Is Collaboration Paying Off for Firms? Business Horizons, 49(1), 61-70.

Departamento Nacional de Planeación. (2007). Agenda Interna para la Productividad y la Competitividad de Sucre. Bogotá.

Espinosa , F. A. (2012). TLC y Carne de Bovino. Carta FEDEGAN. Federación Colombiana de Ganaderos, 142-146.

Estrada, R., \& Holmann, F. (2008). Competitividad de la Producción de Leche frente a los Tratados de Libre Comercio en Nicaragua, Costa Rica y Co- 
lombia. (Documento de Trabajo no. 207). Cali: Co: Centro Internacionald Agricultura Tropical (CIAT), International Livestock Research Institute (ILRI).

FAO \& FEPALE. (2012). Situación de la Lechería en América Latina y el Caribe en 2011, Observatorio de la Cadena Lechera. Ofcina Regional de la FAO para América Latina y el Caribe, División de Producción y Sanidad Animal. Chile.

Faust, V., Christens, B. D., Sparks, S. M., \& Hilgendorf, A. E. (2015). Exploring relationships among organizational capacity, collaboration, and network change. Psychosocial Intervention, 24(3), 125-131.

Fawcett, S. E., Magnan, G. M., \& McCarter, M. W. (2008). Benefits, barriers, and bridges to effective supply chain management. Supply Chain Management: An International Journal, 13(1), 35-48.

FEDEGAN. (2009). Lo que usted necesita saber sobre la leche en Colombia. Bogotá.

FEDEGAN. (2012). Retos de la Globalozación en el Mercado lácteo. Bogotá: Federación Nacional de Ganaderos.

FEDEGAN. (2013). Proyecto de Ley para el sector Lácteo: ¿Qué piden los ganaderos al Gobierno? Carta No. 135, 14-33. Bogotá.

FEDEGAN. (2015). Informe Especial: Importancia de Modernizar las Lecherías. Carta FEDEGAN - Federación Colombiana de Ganaderos, 151, 12-21.

Fisher, M., Hammond, J., Obermeyer, W., \& Raman, A. (1997). Configuring a supply chain to reduce the cost of demand uncertainty. Production and operations management, 6(3), 21l-225.

Fluck, C. (2014). Closing the Gap between the Current and Potential Conditions in the Cocoa (Production) Industry: Comparing Colombia and Mexico. Bachelor`s Thesis, University of Twente, 1-37.

Francois, J., Moad, K., Bourrieres, J. P., \& Lebel, L. (2017). A tactical planning model for collaborative timber transport. IFAC-PapersOnLine, 50(1), 11713-11718.

FUNDACIÓN ALPINA. (2012). Análisis de Mercado. Popayán (Colombia): Aalcaldia municipal de Popayán Y Universidad el Cauca.

Fundesarrollo. (Junio de 2014). La Región Caribe en Cifras. Obtenido de Fundación para el Desarrollo de Caribe: http://www.fundesarrollo.org.co/ wp-content/uploads/2014/06/Regi\%C3\%B3n-Caribe-en-Cifras.pdf 
FUNDESARROLLO. (2014). La Región Caribe en cifras. Fundación para el Desarrollo del Caribe.

Gligor, D. M., \& Holcomb, M. (2013). The role of personal relationships in supply chains: an exploration of buyers and suppliers of logistics services. International Journal of Logistics Management, 24(3), 328-355.

Govindan, K., Soleimani, H., \& Kannan, D. (2014). Reverse logistics and closed-loop supply chain: A comprehensive review to explore the future. European Journal of Operational Researh, 1-57.

Govindan, K., Soleimani, H., \& Kannan, D. (2014). Reverse logistics and closed-loop supply chain: A comprehensive review to explore the future. European Journal of Operational Research, 1 - 57.

Grocery Manufacturers Asocciations. (2005). Manual de la Cadena de Abastecimiento de Productos Alimenticios. Washinton.

Guzman, K. (2013). La industria láctea en Valledupar: primera en la región Caribe [online]. Recuperado el Agosto de 2015, de http://www.banrep.gov.co/docum/Lectura_finanzas/pdf/dtser_184.pdf/

Han , J. H., Wang, Y., \& Naim, M. (2017). Reconceptualization of information technology flexibility for supply chain management: An empirical study. International Journal of production Econimics, 187, 196-215.

Heirati, N., \& Siahtiri, V. (2017). Heirati, N., \& Siahtiri, V. (2017). Driving service innovativeness via collaboration with customers and suppliers: Evidence from business-to-business services. Industrial Marketing Management, .

Hernandez , F., Vertel, M., \&e Porto, I. (2016). Análisis Factorial Múltiple de la competitividad de empresas lácteas del municipio de Sincelejo, Colombia. Agronomía Colombiana, 34(1Supl), S36-S40.

Hernandez, F. D., Bustamante, L. F., \& Porto, I. A. (2014). Evaluación del grado de competitividad de cinco empresas del sector lechero de Sincelejo, Colombia. Revista de Investigación, Desarrollo e Innovación, 5(1), 8-19.

Howeg, M., Disney, S., Holmstrom, J., \& Smaros, J. (2005). Supply chain collaboration:: Making sense of the strategy continuum. European management journal, 23(2), 170-181.

Instituto Colombiano Agropecuario - ICA. (2016). Censo Pecuario Nacional. Bogotá: Ministerio de Agricultura. 
Jaramillo, A., \& Areiza, A. (2012). Market Analysis of Milk and Dairy Products in Colombia (2008-2012). Bogotá: Super Intendencia de Industria y Comercio.

Jiménez, J., \& Hernández, S. (2002). Marco conceptual de la cadena de suministro: un nuevo enfoque logístico. En S. d. Transportes, Publicación Técnica No. 215 (págs. 1-272). Sanfandila, Qro: Instituto Mexicano del Transporte.

Jonkman, J., Bloemhof, J. M., Van der Vorst, J. G., \& Van der Padt, A. (2017). Selecting food process designs from a supply chain perspective. Journal of Food Engineering, 195, 52-60.

Kalwani, M. U., \& Narayandas, N. (1995). Long-term manufacturer-supplier relationships: do they pay off for supplier firms? The Journal of marketing, 1-6.

Kelly, A., Leitner, G., \& Merin, U. (2011). Milk quality and udder health: Test Methods and Standards. Encyclopedia of Dairy Sciences. 2 ed. San Diego (USA), 894 - 901.

Kohli, A. S., \& Jensen, J. B. (2010). Assessing effectiveness of supply chain collaboration: an empirical study. Supply chain forum: An international journal, $11(2), 2-16$.

Kotabe, M., Martin, X., \& Domoto, H. (2003). Gaining from vertical partnerships: knowledge transfer, relationship duration, and supplier performance improvement in the US and Japanese automotive industries. Strategic Management Journal, 24(4), 293-316.

Kumar, G., Banerjee, R. N., Meena, P. L., \& Ganguly, K. K. (2017). Joint planning and problem solving roles in supply chain collaboration. IIMB management review, 29(1), 45-57.

Lee, H. L., Padmanabhan, V., \& Whang, S. (1997). Information distortion in a supply chain: The bullwhip effect. Management Science, 43(4), 546-558.

Lee, H., Kim, M. S., \& Kim, K. K. (2014). Interorganizational information systems visibility and supply chain performance. International Journal of Information Management, 34(2), 285-295.

Li, D., Wang, X., Chan , H., \& Manzini, R. (2014). Sustainable Food Supply Chain Management. International Journal of Production Economics, 152, 1-8.

Liao, S. H., Hu, D. C., \& E Ding, L. W. (2017). Assessing the Influence of Supply Chain Collaboration Value Innovation, Supply Chain Capability and Competitive Advantage in Taiwan's Networking Communication Industry. International Journal o Production Economics, 191, 143-153. 
Liu, C., \& Yao, J. (2018). Dynamic supply chain integration optimization in service mass customization. Computers \& Industrial Engineering, 120, 42-52.

Lombana, J., Martinez, D., Valverde, M., Rubio, J., Castrillón, J., \& Marino, W. (2012). Caracterización del Sector Ganadero del Caribe Colombiano. 1-76.

Martínez, M., \& Gómez, C. (2013). Compositional and Hygienic Quality of Raw Molk Received in Dairies in Sucre, Colombia. Biotecnología en el Sector Agropecuario y Agroindustrial. Vol 11 No. 2, 93 - 100.

Martínez, M., \& Gómez, C. (2013). Compositional and Hygienic Quality of Raw Molk Received in Dairies in Sucre, Colombia. Biotecnología en el Sector Agropecuario y Agroindustrial. Vol 11 No. 2, 93- 100.

Martìnez, M., Serpa, J., \& Gomez, C. (2009). Diagnostico de la calidad Composicional e Higiénico sanitarias de la Leche Cruda en Centros de Acopio y Plantas Procesadoras del Departamento de Sucre. Hipertexto Ltda. Sincelejo, Colombia, 1-10.

Mathuramaytha, C. (2011). Supply chain collaboration-What's an outcome? A theoretical model. International Conference on Financial Management and Economics IPEDR, IACSIT Press, Singapore, 11, 102-108.

Meneses, R., Estrada, D., Chantré, C., \& López, F. (2015). Characterization in the non formal chain of raw millk at the municipality of Popayan. Biotecnología en el Sector Agropecuario y Agroindustrial Vol 13 No. 2 , 130-139.

Miemczyk, J., Johnsen, T., \& Macquet, M. (2012). Sustainable purchasing and supply management: a structured literature review of definitions and measures at the dyad, chain and network levels. Supply Chain Management International Journal, 17(5), 478-496.

Ministerio de transporte. (2010). Plan nacional de desarrollo 2010 - 2014. Sector transporte. Bogotá: ministerio de transporte.

Ministerio de Agricultura y Desarrollo Rural. (2005). La Cadena de lácteos en Colombia. Una mirada global de su estructura y dinámica 1995-2005. Bogotá, Marzo de 2005: Observatorio Agrocadenas de Colombia.

Ministerio de Agricultura y Desarrollo Rural. (2007). Agenda prospectiva de Investigación y Desarrollo Técnológico para la Cadena Láctea Colombiana. Bogotá D.C: Giro Editores Ltda.

Ministerio de Agricultura y Desarrollo Rural. (2010). Dirección de Política Sectorial - Grupo de Análisis Sectorial. Boletín de análisis por producto. boletín No. 6. Bogotá: MADR. 
Ministerio de Trabajo. (2014). Plan Departamental de Empleo de Sucre. Convenio $\mathrm{N}^{\circ}$ 188. Programa de Asistencia Técnica para el Fortalecimiento de las Políticas de Empleo, Emprendimiento y Generación de Ingresos. Fundación Panamericana para el Desarrollo - FUPAD. Opciones Gráficas Editores Ltda.

Ministrerio de Salud y Protección Social. (2011). Decreto Número 1880 de 2011 . Por el cual se señalan los requisitos para la comercialización de leche cruda para consumo humano directo en el territorio nacional. Bogotá.

Moghaddam, M., \& Nof, S. Y. (2016). Real-time optimization and control mechanisms for collaborative demand and capacity sharing. International Journal of Production Economics, 171(4), 495-506.

Mojica, F., Trujillo, R., Castellanos, D., \& Bernal, N. (2007). Agenda prospectiva de investigación y desarrollo tecnológico de la cadena láctea colombiana. Bogotá: Ministerio de Agricultura y Desarrollo Rural. Obtenido de www. minagricultura.gov.co.

Murphy, M., O’Mahony, M., Shalloo, L., French, P., \& Upton, J. (2014). Comparison of modelling techniques for milk-production forecasting. Journal of Dairy Science, 97, 3352-3363.

Olivero, R., Aguas, Y., \& Cury, K. (2011). Comercialización de Leche Cruda en Sincelejo, Sucre, Colombia. Revista Colombiana de Ciencia Animal-RECIA, 3(1), 157-163.

Olivero, R., Aguas, Y., \& Cury, K. (2011). Raw Milk Marketing in Sincelejo, Sucre, Colombia. Rev. Colombiana cienc. Anim. 3(1), 157- 163.

Orjuela, A. (2013). Estudio de la Cadena Láctea y su asporte a la Competitividad de la zona noroccidental del Municipio de Pasto, Departamento de Nariño. San Juan de Pasto.

Perny, J. F., Crump, P. M., Hernandez, L. L., \&e Reinemann, D. J. (2018). Association of milking interval and milk production rate in an automatic milking system. Journal of Dairy Science, 101, 1616-1625.

PROEXPORT. (2011). Sector Lácteo en Colombia. Bogotá, D.C.: PROEXPORT COLOMBIA.

Proexport. (2013). Sucre Aprovecha los TLC. Revista de las Oportunidades Proexport Colombia, 1-24.

Red Nacional de Agencias de Desarrollo Local. (2013). Plan Estrategico Departamental de Ciencia, Tecnología e Innovación de Sucre. Sucre Innova, Sucre 
Transforma - PEDCTI. Convenio 0592-2012. Sincelejo: Gobernación de Sucre.

Red Nacional de Agencias de Desarrollo Local. (2013). Plan Estrategico Departamental de Ciencia, Tecnología e Innovación de Sucre. Sucre Innova, Sucre Transforma. Convenio 0592-2012. Sincelejo: Gobernación de Sucre.

Sana, S. S. (2012). A collaborating inventory model in a supply chain. Economic Modelling, 29(5), 2016-2023.

Secretaria de Desarrollo Económico y Medio Ambiente. (2016). Informe de Coyuntura Departamental. Sincelejo: Gobernación de Sucre.

Shortall, J., Foley, C., Sleator, R. D., \& O'Brien, B. (2018). The effect of dairy cow breed on milk production, cow traffic and milking characteristics in a pasture-based automatic milking system. Livestock Science, 209, 1-7.

Simatupang, T. M., \& Sridharan, R. (2005). The collaboration index: a measure for supply chain collaboration. International Journal of Physical Distribution \& Logistics Management, 35(1), 44-62.

Singh, P. J., \& Power, D. (2009). The nature and effectiveness of collaboration between firms, their customers and suppliers: a supply chain perspective. Supply Chain Management: An International Journal, 14(3), 189-200.

Tsanos, S., Zografos, G. K., \& Harrison, A. (2014). Developing a conceptual model for examining the supply chain relationships between behavioural antecedents of collaboration, integration and performance. The International Journal of Logistics Management, 25(3), 418-462.

Weber, C. A., Current, J., \& Desai, A. (2000). An optimization approach to determining the number of vendors to employ. Supply Chain management: An international journal, 5(2), 90-98.

Wu, I. L., \& Chiu, M. L. (2018). Examining supply chain collaboration with determinants and performance impact: Social capital, justice, and technology use perspectives. International Journal of Information Management, 39, 5-19.

Zhang, F., Murphy, M., Shallo, L., Ruelle, E., \& Upton, J. (2016). An automatic model configuration and optimization system for milk production forecasting. Computers and Electronics in Agriculture, 100-11l.

Zhang, Q., \& Cao, M. (2018). Exploring antecedents of supply chain collaboration: Effects of culture and interorganizational system appropriation. International Journal of Production Economics, 195, 146-157. 